\title{
ABSORPTION SPECTROPHOTOMETRY
}

\author{
D. L. TIMMA \\ Mound Laboratory ${ }^{1}$, Monsanto Chemical Company, Miamisburg, Ohio
}

Absorption spectrophotometry in many ways offers a means of analysis which supplements the information obtainable by emission spectroscopy and allows investigation of whole new classes of materials. Consequently, emission and absorption spectroscopy are closely related and instrumentation for both is quite often found in the same laboratory. Before discussing the specific applications of absorption spectrophotometry one should first know of the origin of absorption spectra, become familiar with the general components of the instruments used for measuring absorption spectra, and understand the laws governing the attenuation of radiant energy passing through a homogeneous, isotropic, non-metallic medium.

\section{ORIGIN OF ABSORPTION SPECTRA}

Emission spectra consist of sharp discrete lines arising from the transition of electrons from a term level of higher energy to a term level of lower energy in an atom or ion. These term levels can be defined by a principle quantum number, $n$; an azimuthal quantum number, 1 ; a spin quantum number, s; and the resultant angular momentum, $j$. The term levels of molecules are designated by a similar system of symbols where $\Lambda$ is analogous to the azimuthal quantum number, $\Sigma$ the spin quantum number, and $\Omega$ is similar to the resultant angular momentum. In emission spectroscopy the electrons are normally "excited" to the higher energy levels by energy supplied by means of electrical discharge. The amount of energy absorbed in this process is small and is not normally measured, since it is the energy emitted by the excited atoms which serves to qualitatively identify the atoms and to serve for their quantitative estimation.

Absorption spectroscopy is a means of investigating samples by the energy which is absorbed in raising its molecules to excited states. While the phenomenon

${ }^{1}$ Operated by the Monsanto Chemical Company, under AEC Contract Number AT-33-1-GEN-53.

The Ohio Journal of Science 52(3): 117, May, 1952. 
of energy absorption is observed for atoms and ions, this method of analysis is more generally applied to the determination of molecules or particular groups of atoms within a molecule. Since it is made up of several atoms, the energy of a molecule is the sum of the translational energy of the entire system, the vibrational energy, the rotational energy, and the electronic energy of its components. Consequently, it is found that the energy terms for molecules are more complicated than for atoms, and each spectral line of the atomic emission spectrum is replaced by a system of bands. Energy transitions which involve change of rotational energy alone involve relatively small amounts of energy and correspond to bands in the extreme infrared or microwave regions. The vibrational energies are larger so that changes in vibrational (and rotational) energy lead to bands in the near infrared. Transitions involving electronic rearrangement in molecules may lead to absorption of energy in any region of the spectrum. The systems normally used for analytical spectrophotometry absorb energy between $2,000 \mathrm{~A}$ and $10,000 \mathrm{~A}$. In general, the region of the spectrum in which an absorption band occurs is determined by the electronic transition, the gross structure of the system depends on vibrational energy changes, and the fine-line structure of the individual bands depends on changes in rotational energy.

\section{INSTRUMENTATION}

Since qualitative spectrophotometry involves measurement of the energy absorbed by a sample as a function of the wavelength and quantitative determinations depend upon the measurement of the absorbed energy at a fixed wavelength, a spectrophotometer must consist of three essential components. These parts are a source of radiant energy, a means of dispersing the energy according to its frequency, and a mechanism for detecting and estimating the amount of energy passing through the sample.

In the ultraviolet and visible portions of the electromagnetic spectrum, three types of sources of radiation are normally used. In the ultraviolet region, from 200 millimicrons to 400 millimicrons, the continuum produced by a hydrogen discharge has been widely used (Lawrence and Edlefson, 1930; Kistiakowsky, 1931). This source gives a continuous source of radiation below about 350 millimicrons and is readily adaptable for use with instruments that contain phototubes or photomultiplier tubes as radiation detectors. Such sources are operated at hydrogen pressures ranging from 1 to 10 millimeters of mercury, at applied voltages from 3,000 to 5,000 volts, and at a fraction of an ampere to several amperes. A hot-cathode, hydrogen-discharge tube has been described (Allen and Franklin, 1939,1941 ) which operates at 80 volts and about 1.3 amperes. A ribbon-filament incandescent lamp operating at a color temperature of $2,400^{\circ}$ to $2,800^{\circ} \mathrm{K}$ gives a continuous source of radiation which is usable down to about 320 millimicrons. This limit is imposed by the glass envelope surrounding the filament. A condensed spark between tungsten-steel electrodes is the most widely used source in photographic absorption spectrophotometry. With these electrodes the high intensity of the ultraviolet emission, the richness in lines and their fairly even distribution over the spectral range, the freedom from wandering, and the localization of the luminiferous area in a small space make this source preferred for work in the ultraviolet region. The discontinuous character of the spectrum and the wide range of intensities between the strong and weak lines are disadvantages of this source. These two properties make the spectral image unsuited to the detection of fine structure in absorption bands.

Spectral dispersion and isolation in the region between 200 and 1,000 millimicrons may be obtained by means of refraction through a prism; by the diffraction and interference associated with a fine-line grating; or the spectral isolation may be effected by means of optical filters, there being no dispersing system in the ordinary sense. 
Glass or quartz prisms of $60^{\circ}$ refracting angle, or equivalent prisms, have been widely used in prism spectrophotometers for the ultraviolet and visible regions. Recently a spectrophotometer has become available which uses a Féry prism. Some instruments have been designed with a double-prism monochromator to reduce stray light.

Gratings have been used to obtain dispersion of the radiant energy, with both transmission and reflection types being of value. A transmission-grating, doublemonochromator spectrophotometer is claimed to have an average stray radiation of less than one percent. However, in general, a grating monochromator has a higher relative intensity of stray radiation than a prism dispersing system (Cary and Beckman, 1941).

In certain kinds of work where it has been found adequate to isolate only certain spectral regions, optical filters have proven to be satisfactory. These filters are of three general types: (1) glasses, usually two or more in combination, (2) two or more dyes incorporated into gelatin or other medium, and (3) interference filters. Information on the characteristics of these filtering media may be obtained from the Corning Glass Works (Glass Color Filters, 1946), the Eastman Kodak Company supplying the Wratten dyed-gelatin filters (Wratten Light Filters, 1938), and the Farrand Optical Company (Farrand Interference Filters, 1947) and Baird Associates (Baird Associates Interference Filters, 1946) supplying interference filters.

Spectrophotometry inherently consists of measuring the ratio of two radiant energies at a specified frequency or wavelength, and then repeating this measurement at other frequencies or wavelengths as often as desired over the spectral range of interest. This ratio of radiant energies may be determined by visual, photographic, or photoelectric means. In visual spectrophotometry, the photometric part of the instrument includes a two-part photometric field and a means of varying the luminance of one of the parts so that the eye is used only to detect unmatch and finally to judge match of the two parts of the field. The means of varying the luminance of one part of the field is calibrated, so that the actual value of the match point is determined by this auxiliary system. Photographic photometry is normally used when a conventional spectrograph is used as a dispersing medium. Evaluation of the spectra requires a previous calibration of the photographic emulsion, much in the manner required for quantitative emission spectroscopy. However, because of the higher dispersion normally associated with spectrographs, more fine structure may be investigated by this technique than can be observed visually or with photoelectric photometers. Photoelectric detectors are of three general types: (1) barrier layer cells, (2) phototubes, and (3) photomultiplier tubes. A barrier layer cell consists essentially of a plate of either copper or iron upon which a semiconducting layer of cuprous oxide or selenium has been grown. The semiconducting layer is covered with a light-transparent layer of gold, platinum, copper, or lead which acts as an electron collector. The average cell has an output of about 120 microamperes per lumen and can be used in conjunction with microor milli-ammeters to detect and measure high levels of illumination. No external source of potential is required with a barrier layer cell. Vacuum phototubes require a source of potential and are normally used with a vacuum tube amplifier. Over the range of intensities encountered in spectrophotometry, they give a linear response. A special kind of phototube employing several electrodes or dynodes which achieves the equivalent of amplification within the tube itself is called a photomultiplier tube. These tubes have a higher signal-to-noise ratio than the phototubes-amplifier combination.

\section{SPECTROPHOTOMETRIC IDENTIFICATION}

Qualitative absorption spectrophotometry may be achieved by comparing the absorption curve of the unknown with the curves of known compounds. 
Accurate data on the absorption of a sample as a function of wavelength must be obtained over a considerable portion of the spectrum to allow this method to be used. Similar structures within different molecules will show similar absorptions, and, likewise, similar complex organic molecules will show very similar absorption spectra. In fact, this generalization has been so well established the tables of the wavelengths of the absorptions of different groups in the ultraviolet and visible regions have been made (Harrison, Lord, and Loofbourow, 1948). This general technique has been used to identify the fragments obtained by treating natural vitamin $B_{1}$ with sodium sulfite, thus facilitating the synthesis of thiamine (Williams and Ruehle, 1937). This is an empirical method and depends upon having a library of absorption curves or a clue to the composition of the sample so that similar compounds may be prepared.

Unfortunately no single comprehensive compilation of absorption spectra exists. Some data have been compiled by the American Petroleum Institute Research Project 44 at the National Bureau of Standards and is available as the Catalog of U1traviolet Spectrograms. International Critical Tables contain both absorption data and references to literature containing absorption spectra data on both organic and inorganic compounds. "Tables annuelles de constantes et données numeriques, données numeriques de spectroscopie," Vols. I to XII by V. Henri and L. Bruninghaus contain tabulations of absorption data. Landolt-Bornstein, "PhysikalischeChemische Tabellen," edited by W. A. Roth and K. Scheel; Beilstein, "Handbuch der Organischen Chemie," 4th edition; and Gmelin, "Handbuch der Inorganischen Chemie," 8th edition all contain literature references to absorption spectra. Brode (1945) has made a critical evaluation of most of the available collections of data.

\section{QUANTITATIVE SPECTROPHOTOMETRY}

For quantitative determinations a more precise knowledge of the influence of different variables upon the absorption of energy is required. The simplest case of absorption is that in which a parallel beam of monochromatic radiation passes rectilinearly through a homogeneous absorbing medium. Under these conditions the intensity of the radiation is reduced by the same fractional part by each succeeding portion of its path. Thus, if the intensity is reduced by one third in the first millimeter, it will be reduced to one ninth of its original intensity by the second millimeter. This fact was first enunciated by Bouguer in 1729. It was later applied by Lambert and is sometimes known as Lambert's Law. When dealing with solutions, in which molecules are acting as energy absorbers, the optical path may be varied either by changing the cell thickness (Lambert's Law) or by changing concentration of the solute. The latter method, Beer's Law, assumes that the specific absorption per molecule of the absorbing material is independent of its concentration in the solution. These laws are combined in the familiar expression $\mathrm{E}=\mathrm{kcd}$ where $\mathrm{k}$ is the specific absorption coefficient, $\mathrm{c}$ is the concentration, and $d$ is the sample thickness. As observed in the use of this technique for qualitative analysis, the extinction of a sample is a function of the wavelength, so quantitative absorption spectrophotometry consists of measuring the absorbancy of sample at a wavelength, or a narrow range of wavelengths, and correlating the extinction with concentration. Quantitative determinations involve merely the solving of simple algebraic equations if the absorbancies of the components of a solution are additive. However, if absorbancies are not additive, quantitative determinations become empirical and depend upon the previous determination of a working curve showing the absorbancy-concentration relationship.

While many instances are known where Beer's Law is said to be valid, deviations are both frequent and significant. Körtum (1936) has stated that "the validity of Beer's Law for ions must be considered as an exception rather than as a rule. Validity over large concentration ranges is not to be expected since Beer's Law is a limit law for low concentrations." The deviations from Beer's Law are of two types - chemical and physical or instrumental. 
It is generally concluded that the observed deviations are more a result of chemical changes affecting or occurring in the absorbing ions than in a failure of the law (von Halban and Ebert, 1924). Körtum and Seiler (1939) have summarized the causes for divergence from the law. According to the theory of light dispersion, it is not the specific absorption coefficient $\mathrm{k}$ but the quantity $\mathrm{kn} /\left(\mathrm{n}^{2}+2\right)^{2}$ which is a constant, independent of concentration. For concentrations lower than 0.01 molar the change in refractive index, $\mathrm{n}$, would rarely cause an error greater than the accuracy of the measurement. Many organic materials have weak acidic properties, and their equilibria are easily disturbed by changes in acidity. For this reason it is often necessary to control the $\mathrm{pH}$ of the solution within narrow limits to obtain reproducible results. Equilibria may also be displaced by the presence of non-absorbing materials in the sample through the mass action law. Temperature fluctuations may influence absorption data in two different manners. It may cause a shift in the equilibrium constant of the absorbing system, and it may cause concentration changes due to volume changes caused by expansion. The magnitude of the effect of temperature change depends upon the material. It amounts to 0.1 percent per degree at 436 millimicrons for chromate ion and 1 percent per degree for 2, 4-dinitrophenolate ion (Körtum and von Halban, 1934).

What may be attributed to a deviation from Beer's Law may be a low degree of monochromaticity of the radiant energy used. Even the best monochromators give a spectral band of finite width, and apparent deviations will be noted if the absorbancy of the sample changes considerably across the spectral interval isolated by the monochromator (Brattain, Rasmussen, and Cravath, 1943). Poor matching of absorption cells and stray radiation may also be factors.

\section{TREATMENT OF DATA}

Qualitative data are generally presented as curves showing the absorptivity as a function of the wavelength through the spectral range covered. No single method of presentation of this data has become universally accepted. In fact, Mellon (1949) has said "the confusion in usage of terms, symbols, and data in absorptiometry remains unsurpassed by that in any other division of quantitative analysis." The ordinate is usually plotted in increasing values of transmittance, $\mathrm{T}_{i}$, absorbancy, $\mathrm{A}_{\mathrm{s}}$, (also known as optical density, $\mathrm{D}$, or extinction, $\mathrm{E}$ ), extinction coefficient, $k$, (or absorbancy index, $a_{s}$ ), molecular extinction, $\epsilon$, (or molar absorbancy index, $a_{m}$ ) or the logarithm of any of them. The abscissa is used to present the region of spectrum investigated and may be expressed in one or more of the following terms: wavelength, $\lambda$, (in millimicrons $m \mu$, or Angstroms, A); frequency, $\nu$ (in fresnels); wavenumber, $\nu^{\prime}$ (in waves per centimeter); or the logarithm of the wavelength. Wavelength, wavenumber, and frequency are related by the expression

$$
\frac{1}{\text { Wavelength }}=\text { Wavenumber }=\frac{\text { Frequency }}{\text { Speed of Light }}
$$

With all possible variations in use, including inconsistency in direction of plotting, and disregard for general good practice in graphing, the literature is likely to be less than clear to anyone unskilled in mentally transforming the curve of unfamiliar form into the form with which he ordinarily deals. Transmittance is often obtained directly from the instruments and therefore used because of convenience. Plotting on a logarithmic scale magnifies the low transmittance values relative to the higher values. Optical density, extinction, or absorbancy magnify the absorption maxima and also facilitate calculation of thicknesses, since the relationship is linear if the solution conforms to Beer's Law.

Quantitative absorption spectrophotometry depends upon the correlation of the amount of energy absorbed at a given frequency with the amount of material 
present in the sample. This correlation is known as a calibration or a working curve and consists of plotting transmittance, absorbance, or their logarithms as a function of concentration. Since absorbancy is a linear function of concentration for solutions conforming to Beer's Law, a straight line indicates such conformity. Ringbom (1939) has suggested plotting working curves as absorbancy vs. the logarithm of concentration to emphasize the importance of making measurements within certain transmittancy ranges where the error in measurement is smallest. Ayres (1939) has recently reviewed this technique and pointed out that the minimum error in absorbancy determination occurs at about 28 percent transmission.

\section{USES OF ABSORPTION SPECTROPHOTOMETRY}

The chief justification for the development of equipment or methods is the possibility of using them for the determination of the constituents of a sample, or the measurement of properties of systems. Since anions, cations, inorganic groups, organic radicals, and whole molecules all produce systems which are capable of causing the absorption of energy in the ultraviolet and visual regions, the uses of absorption spectrophotometry are so numerous that only the general aspects of the methods and the general fields of applications can be discussed here. For a more detailed discussion of individual applications to analytical problems, some of the standard texts on absorption spectrophotometry should be consulted (Brode, 1945; Snell and Snell, 1948; Welcher, 1947; Mellon, 1950). Reviews of current progress in this field have been published annually for the last three years in the January issues of Analytical Chemistry and present bibliographies to most of the work published in American journals during the year.

Absorption spectroscopy has been applied to problems of determining the isomeric composition of a sample whose constituents are otherwise chemically identical. The analysis of xylene isomers has been discussed by Shostenko and Shtandel (1948). Stair (1949) has discussed the analytical value of the absorption bands in the absorption spectra of four butylbenzenes and three diethylbenzenes.

Complexes which are formed in solution between metals and organic reagents, two organic materials, or two inorganic radicals may be studied by means of absorption spectrophotometry. This method has been used by Molland (1940) to identify the iron complex of 8-hydroxyquinoline-5-sulfonic acid as $\left(\mathrm{C}_{9} \mathrm{H}_{6} \mathrm{O}_{4} \mathrm{NS}\right)_{3} \mathrm{Fe}$. Evidence for the complex ion $\mathrm{Fe}(\mathrm{CNS})^{++}$was obtained by photometric means (Bent and French, 1941) and later substantiated by migration experiments.

Because of the speed with which measurements can be taken, and more recently because of the development of automatic recording instruments, spectrophotometric analysis is adaptable to the investigation of unstable systems. Probably one of the most fugitive color reactions used for identification is the antimony trichloride-Vitamin A complex where the time of reaction and measurement must be carefully controlled. Readings are normally taken from $\tilde{5}$ to 30 seconds after adding the reagent, procedures varying among laboratories. A method for the determination of ascorbic acid based on the decolorization of 2, 6-dichlorophenolindophenol (Hochberg, Melnick, and Oser, 1943) requires that readings be taken at 5 and 10 seconds while the reduction of the dye is proceeding. Gibson and Taylor (1945) have devised a method by which the absorption spectrum can be observed as the sample material flows through the absorption cell. By adjusting the rate of flow one can investigate a system at any predetermined time after mixing the components.

\section{CONCLUSIONS}

Given a suitable reaction, a spectrophotometric method can be developed if the intensity of the coloration produced is essentially dependent upon the concentration of the desired constituent, and is stable long enough to permit measurement. The development of specific organic reagents and effective means of complexation 
have made possible the determination of single components found in complex systems. Spectrophotometric methods, not being limited to elements, can often be applied to the determination of compounds, radicals, and functional groups. Furthermore, chemical phenomena which occur in solution, as complex formation, solvation, and equilibria, may be studied in situ by spectrophotometric methods. Absorption spectrophotometry thus provides a useful analytical and research tool which bridges the span between the data on atomic and ionic species supplied by emission spectroscopy and the information on molecular and crystalline states obtained from X-ray diffraction.

\section{LITERATURE CITED}

Allen, A. J., and R. G. Franklin. 1939. Jour. Optical Soc. Amer., 29: 453.

Allen, A. J., and R. G. Franklin. 1941. Jour. Optical Soc. Amer., 31: 268.

Ayres, G. H. 1939. Ind. Eng. Chem., Anal. Ed., 11: 72.

Baird Associates, Inc. 1946. "Baird Associates Interference Filters.'

Bent, H. E., and C. L. French. 1941. Jour. Amer. Chem. Soc., 63: 568.

Brattain, R. R., R. S. Rasmussen, and A. M. Cravath. 1943. Jour. Applied Phys., 14: 418.

Brode, W. R. 1945. Chemical Spectroscopy. John Wiley \& Sons, New York, N. Y.

Cary, H. H., and A. O. Beckman. 1941. Jour. Optical Soc. Amer., 31: 682.

Corning Glass works. 1946. "Glass Color Filters."

Eastman Kodak Company. 1938. "Wratten Light Filters."

Farrand Optical Company. 1947. "Farrand Interference Filters."

Gibson, G. P., and R. J. Taylor. 1945. Analyst, 70: 449.

Harrison, Lord, and Loofbourow. 1948. Practical Spectroscopy, p. 275. Prentice Hall, New York, N. Y.

Hochberg, M., D. Melnick, and B. L. Oser. 1943. Ind. Eng. Chem., Anal. Ed., 15: 182.

Kistiakowsky, G. B. 1931. Rev. Sci. Inst., 2: 549.

Körtum, G. 1936. Z. physik. Chem., B34: 255.

Körtum, G., and M. Seiler. 1939. Angew. Chem., 52: 687.

Körtum, G., and H. von Halban. 1934. Z. physik. Chem., A170: 212.

Lawrence, E. O., and N. E. Edlefson. 1930. Rev. Sci. Inst., 1: 45.

Mellon, M. G. 1949. Anal Chem., 21:3.

Mellon M. G. 1950. Analytical Äbsorption Spectroscopy. John Wiley \& Sons, New York, N. Y.

Molland, J. 1940. Jour. Amer. Chem. Soc., $62: 541$.

Ringbom, A. 1939 . Z. anal Chem., 115: 332.

Shostenko, Y. V., and A. E. Shtandel. 1948. Zhur. Priklad. Khim., 21: 408.

Snell, F. D., and C. T. Snell. 1948. Colorimetric Methods of Analysis. D. Von Nostrand Co., New York, N. Y.

Stair, R. $1949 . \quad$ Jour. Research Natl. Bur. Standards, 42: 587.

von Halban, H., and L. Ebert. 1924. Z. physik. Chem., 112: 321.

Welcher, F. J. 1947. Organic Analytical Reagents. D. Van Nostrand Co., New York, N. Y. Williams, R. R., and A. E. Ruehle. 1937. Jour. Amer. Chem. Soc., 59: 526-30, 530-33, $1052-54$. 